\title{
POWER SYSTEM OSCILLATION DAMPING CONTROL BY ROBUST SVC SUPPLEMENTARY CONTROLLER
}

\author{
Ferdi Armansyah, Student Member (Hiroshima University) \\ Naoto Yorino, Member (Hiroshima University) \\ Hiroshi Sasaki, Member (Hiroshima University)
}

\begin{abstract}
An improved robust $\mathrm{H}_{\infty}$-optimization techniques is used to design a robust supplementary controller for the Static Var Compensator (SVC) to increase the power system damping. The existing $\mathrm{H}_{\infty}$-approach for designing supplementary controller has limitation for the cancellation of the plant's poorly damped poles by the controller's zeros. In this case, supplementary controller designed with the standard $\mathrm{H}_{\infty}$-approach is unable to increase the damping of the lightly damped modes and thereby affect the performance and robustness of the system. Therefore, the bilinear transform has been used in the design to prevent the pole-zero cancellation of the poorly damped poles and to improve the control system performance. The model variations, because of the system operating condition variations, are treated as model uncertainties and are taken into consideration at the controller design stage. The magnitude of the current in the transmission line, $I_{m}$, is chosen as feedback signal. The performance of the controller has been evaluated extensively by non-linear simulation. The result have indicated that the designed controller can provide sufficient damping to the system under a wide range of operating conditions.
\end{abstract}

Key words: low-frequency oscillations, $\mathrm{H}_{\infty_{o}}$-optimal control, SVC, performance, robustness

\section{Introduction}

By introducing a supplemental signal to the voltage setpoint, Static Var Compensator (SVC) supplementary controller can be used to damp low-frequency oscillations in interconnected power systems [1,9]. Several papers have been published on this topic, for example properly tuned, conventional SVC supplementary controller [1], linear optimal control -approach from the modern control techniques [2]. These methods have been shown to be very effective in damping low frequency oscillations. This technique, however, has some limitations. One of its principal disadvantages is lack of robustness. They can not guarantee the stability and the performance of the system under a wide range of operating conditions in spite of uncertainties in the system models.

As another effort, application of adaptive control to power system are also being extensively studied. It has been shown that adaptive control algorithm is more suitable for power systems [3], because they can adapt themselves to changes in system parameter to a larger extent. Nevertheless, in the presence of unmodeled highfrequency dynamics and unmeasurable output disturbances, even such controllers can cause closed-loop instability.

Recently, $\mathrm{H}_{\infty}$-approach has been used to design supplementary controller of SVC. The main advantage of this methodology is that it presents a natural tool for successfully modeling plant uncertainties. Design approaches for the $\mathrm{H}_{\infty}$ optimal control theory have also been proposed in various ways. References [6] and [7] shown design approach based on a Riccati equation approach, as state space feedback solutions to $H_{\infty}$ control problem, where LQ regulator designs relating to the $\mathrm{H}_{\infty}$ performance bounds have been established. Althouht it can give good results there are still disadvantages due to complexity of feedback signals and also problem for measurement of state variables.

It has been shown in [8] based on output feedback, a reformulated mixed sensitivity problem to take into account the disturbances introduced at the plant input have been proposed. The Robust $\mathrm{H}_{\infty}$ supplementary controller of SVC can maintain the stability and the performance of the system for a wide range of operating conditions in spite of uncertainties in the system. However the issues concerning the pole-zero cancellation inheren to the standard $\mathrm{H}_{\infty}$-approach have not been addressed.

To cope with the pole-zero cancellation problem, references [9] and [10] have proposed a robust pole assignment method. In term damping, this method can give good result However, the optimal tuning of the controller is sometimes difficult and likely to produce non-minimum phase controllers (controllers with zeros in the right-half plane). This will ultimately limit the ability of the system to recover during large disturbances such as faults. In $[11,12]$, to prevent this undesirable phenomenon, Folly K.A. et al has proposed a special case of the bilinear transform with two parameter to provide sufficient damping to the system and prevent non minimum phase controllers.

This paper presents the synthesis of $\mathrm{H}_{\infty}$-SVC supplementary controller, where the uncertainties are described using a combination of additive and multiplicative uncertainty representation. In designing supplementary controller of SVC the application of the bilinear transformation is investigated to the plant model to prevent the pole zero cancellation that arise in the designed process. The proposed $\mathrm{H}_{\infty}$-SVC supplementary controller uses the same $\mathrm{H}_{\infty}$-optimization control technique as in reference[11]. Since there have been already succesfully applied to PSS, we applied it for designing the supplementary controller for SVC. From following point of view, there is much difference in the design philosophy between designing PSS and SVC supplementary controller. In power systems, most of the tracking problems are duty of primary controllers. In this case, SVC is installed for controlling the bus voltage at a desired level. In these situations, a supplementary controller is used to increase system damping. Since the objective of the supplementary controller is to damp out system oscillations in a particular frequency band, the design emphasis is different from that of tracking a reference input signal. Therefore, in SVC supplementary controller design, it has to be taken into account that the damping controller should not detract from the primary function of the SVC, such that the bus voltage fluctuation 
is within the desired limit. The control limits are not proper uncertainties. However, it is desirable that the control output be between these limits. In order to satisfy this condition, the control limits are modeled as a performance weighting function. Simulation results show that this techniques has improved the performances of the system for both the transient and the steady state stability.

\section{System Description}

In this paper as shown in figure 1 , we consider a synchronous generator to an infinite bus over a long distance transmission line which is compensated at its midpoint by a Statis Var Compensator (SVC), or Fixed Capacitor - Thyristor Controlled Reactor (FCTCR) type Static Var System(SVS). A PI ( Proportional-Integral) controller is used for the voltage regulation at the high voltage side of the SVC bus. The illustrative block diagram of the system is given in figure $2 a$ and $2 b$. Where $B_{c}$ is TCR susceptance corresponds to the thyristor firing angle $\alpha_{o}, \Delta B$ is the variation in TCR susceptance, $K_{r}$ and $T_{r}$ are SVC main controller parameter, $\Delta V_{F}$ represents the incremental auxiliary signal, $\Delta V_{b u s S V C}$ is the terminal voltage perturbation.

This system representation itself contains many uncertainties, because the network is approximated as an infinite bus. In this study system, the effect of the neglected dynamics at the infinite bus is treated as a disturbance to the plant. The entire system can be expressed in the following stste space form:

$$
\begin{aligned}
& \dot{x}=A_{o} x+B_{o} u \\
& y=C_{o} x+D_{o} u
\end{aligned}
$$

where $x$ is the state variable vector, $r$ is the system input, and $y$ is the output. In the case studied, the input is the reference voltage at the SVC bus, and the output is the magnitude of the current in the transmission line, $I_{m}$

The state equation concerning eq. (1) is that of generator and Static Compensator. For design process, the Park two-axis model of the synchronous machine, is used to describe the generator dynamic. Elements of the generator state vector are $\mathrm{x}_{\mathrm{g}}{ }^{\mathrm{T}}=\left[\Delta \omega, \Delta \delta, \Delta \mathrm{E}_{\mathrm{d}}\right.$, $\left., \Delta \mathrm{E}_{\mathrm{q}}^{\prime}, \Delta \mathrm{E}_{\mathrm{fd}}, \Delta \mathrm{T}_{\mathrm{m}}\right]^{\mathrm{T}} . \omega$ denotes the shaft speed, $\delta$ is the load angle, $E_{d}^{\prime} E^{\prime}$ are the internal machine voltage, $E_{\text {fid }}$ is the field voltage, and $T_{m}^{q}$ is the mechanical torque. And the state equation of the Static compensator axis currents are the output. The state vector corresponding to the static compensator module is $x_{c}{ }^{\mathrm{T}}=$ $\left[\Delta \mathrm{i}_{\mathrm{c}}, \Delta \mathrm{B}\right]^{\mathrm{T}} . \Delta \mathrm{i}_{\mathrm{c}}$ denotes the current through the Static Compensator, $\Delta B$ is the variation in TCR susceptance.

For verification process, the dynamic behavior of the synchronous machine is described by $7^{\text {th }}$ order model, which the axis-currents have been chosen as state variables (see Appendix B). In the design stage, the synchronous machine is represented by simplified model, because by using the $\mathrm{H}$ control optimization, the difference between the simplified and the higher order model can be assumed as uncertainties.

The transfer function of the nominal system is given by:

$$
G_{o}(s)=C_{o}\left(I s-A_{o}\right)^{-1} B_{o}+D_{o}
$$

In power systems, most of the tracking problems are duty of primary controllers. In this case, SVC is installed for controlling the bus voltage at a desired level. In these situations, a supplementary controller is used to increase system damping. Since the objective of the supplementary controller is to damp out system oscillations

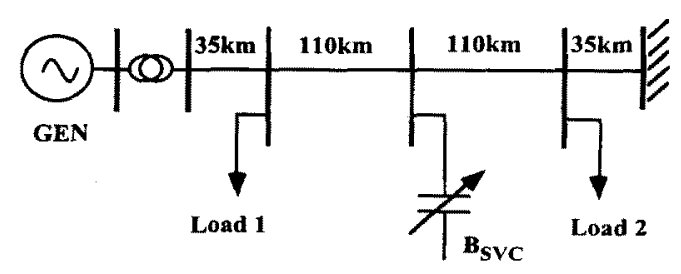

Fig. 1 System Configuration

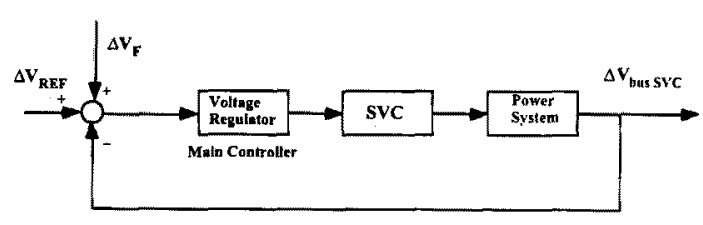

Fig. 2a Block Diagram of The Entire System

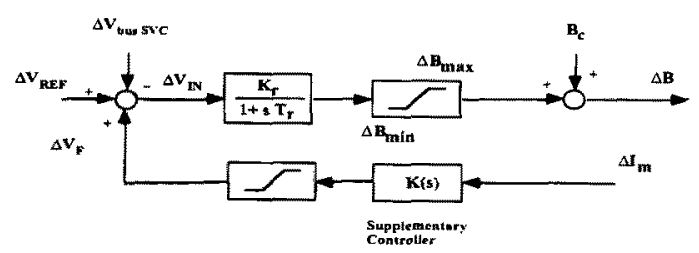

Fig. 2b Block Diagram of the SVC Controller

in a particular frequency band, the design emphasis is different from that of tracking a reference input signal. Therefore, a supplementary controller is needed to provide additional system damping. The block diagram representation of such a control system is shown in figure 3 .

The "plant" $G(s)$ represents the system configuration in Fig. 1 and the block diagram of the entire transfer function shown in Fig. 2a. The output $y(s)$ is the feedback signal of the supplementary controller. In this paper, $y(s)$ is chosen to be magnitude of the line current, $\boldsymbol{I}_{p s}, \boldsymbol{K}(s)$ represents the damping controller to be designed. Signal $d(s)$ is a disturbance acting on the system output $y(s), u$ and $u_{1}$ are the plant input and output, respectively.

To design the proposed SVC Supplementary Controller, first the system is considered under various operating conditions, so that different model $G_{i}(s)$ are obtained. These models include all

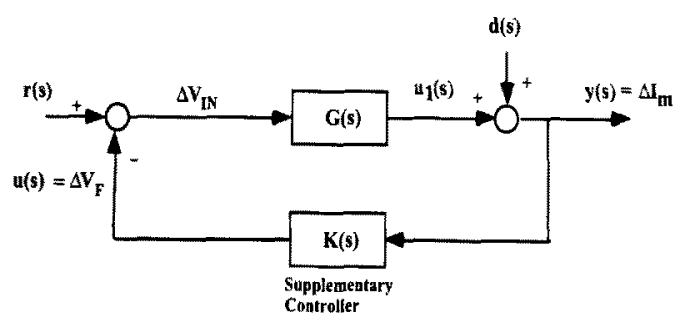

Fig. 3 The plant and the supplementary controller. 
possible representations of the original system. Second, the model uncertainties are derived and the upper bounds are found on these uncertainties. Finally, a controller of single input single output is designed to guarantee the stability of all plant in this set.

\section{Damping Control based on $\mathbf{H}_{\infty}$-optimization theory}

$\mathrm{H}_{\infty}$-optimization of control system deals with the minimization of the peak value of certain closed-loop frequency response functions. In other words, by using the $\mathrm{H}_{\infty}$-control, we wish to design a controller that offers the best protection against the worst case disturbance. That is, a controller that can take into account both robustness and performance specifications.

From figure 3, it is known that the 'true' plant $G(s)$ lies within a set of plants containing the nominal plant $G_{o}(s)$. Therefore, it is common practice to lump the effect of all possible uncertainties into additive and/or multiplicative uncertainties, $W_{2} \Delta_{A}$ and $W_{3} \Delta_{m}$ $\mathrm{W}_{2}$ and $\mathrm{W}_{3}$ are fixed weighting function containing all the information available about the frequency distribution of the uncertainty. $\Delta_{\mathrm{A}}$ and $\Delta_{\mathrm{m}}$ are stable transfer functions representing model additive and multiplicative uncertainty, respectively. $W_{2}$ and $W_{3}$ are chosen such $\|\Delta(s)\|_{\infty} \leq 1$, which implis that Bode plots of $\boldsymbol{W}_{2}$ and $\boldsymbol{W}_{3}$ cover the Bode plots of all possible plants.

Some common issues in the standard $\mathrm{H}_{\mathrm{os}}$-approach is concerned with the following issues:

(i) Disturbance attenuation characteristic is given by

$$
y=S(s) . d
$$

where $S(s)$ is sensitivity function defined by

$$
S(s)=\left(I+K(s) G_{a}(s)\right)^{-t}
$$

(ii) For any pertubation of $\Delta(s), a \Delta(s)$ a $\quad 1$, stability is guaranteed if

$$
\left\|W_{3}(s) T(s)\right\|_{\infty} \leq 1
$$

where a. a denotes the maximum magnitude of the vector over all.$T(s)$ is complementary sensitivity function defined by $T(s)=\left(I+K(s) G_{0}(s)\right)^{-1} K(s) G_{0}(s)$ or $T(S)=I-S(s)$. And also if

$$
\left\|W_{2}(s) R(s)\right\|_{\infty} \leq 1
$$

where $R(s)$ is control sensitivity function defined

by $R(s)=\left(I+C(s) G_{\imath}(s)\right)^{-I} C(s)$.

From (i), disturbance are better attenuated for smaller $S$. The above (ii) prescribe the maximum size of allowable perturbation $W_{2} \Delta_{A}$ and $W_{3} \Delta_{m}$ for stability, implying that for a given $W_{2} \Delta_{A}$ and $W_{3} \Delta_{m}$, larger degree of robust stability is obtain for a smaller $T$ and $R$.

In $\mathrm{H}_{\infty}$-approach, the robust stability goal is met the minimizing for instance the complementary sensitivity function $T(s)$ for the multiplicative uncertainty representation and the control sensitivity function $R(s)$ for the additive uncertainty representation. On the other hand, the nominal performance design goal such as disturbance rejection are achieved by minimizing the $\mathrm{H}_{\infty}$-norm of the sensitivity function $S(s)$. Such conditions can be satisfied by

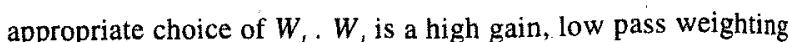
function representing the desired disturbance attenuation.

\section{The Mixed Sensitivity Design}

The design of the SVC-supplementary controller in this paper based on the mixed sensitivity formulation, where the optimization involves the sensitivity function $S(s)$, control sensitivity function $R(s$, and the complementary sensitivity function $T(s):=1-S(s)$. Since $S(s)$ and $T(s)$ can not be minimized simultaneously, frequency dependent weighting function $\left(W_{1}, W_{2}, W_{3}\right)$ are used to guide the design so that a trade-of can be obtained. The performance objective is achieved by minimizing the $S(s)$ in the low frequency ranges where low-frequency disturbances are important. On the other hand, the robust stability is guaranteed by minimizing $R(s)$ and $T(s)$ at high frequency.

Furthermore, the SVC supplementary controller should also be designed such that it does not introduce excessive variations, so that the fluctuation of system variables, such as voltage, transferred power etc., are within desired limits. Such conditions can be satisfied by appropriate choice of $W_{2}$. Based on that $R(s)$ is related to $T(s)$ by $T(s):=G_{o}(s) R(s)$, if the loop gain is large $\left(G_{o}(s) K(s)>>I\right)$ the control sensitivity matrix $R(s)$ approximately equals the inverse $G_{0}(s)^{-1}$ of the plant transfer matrix. Thus, by minimizing $R(s)$, one can limit the plant input, i.e., the controller output. It is also known that by appropriate choice of $W_{2}$, one can also reduce or eliminate the influence of disturbance, i.e., as a performance weighting function. This is achieved by the relation $R(s):=K(s) S(s)$. The weight on the control output $W$, should be chosen close to a differentiator to penalize fast changes and large overshoot in the control output.

The controller is the found by solving the following mixed sensitivity problem (eq. 6) and the feedback loop will take the form as shown in figure 4 .

$$
\min \left\|\begin{array}{l}
W_{1}(s) S(s) \\
W_{2}(s) R(s) \\
W_{3}(s) T(s)
\end{array}\right\|_{\infty}
$$

\section{The Pole-Zero Cancellation Phenomenon.}

The Pole-Zero Cancellation Phenomenon associated with this $\mathrm{H}_{\infty}$-approach [4] makes it impossible for the controller to increase the damping of poorly damped modes. Namely, The $\mathrm{H}_{\mathrm{or}}$ controller always cancel the stable poles of the plant with its transmission zeros (pole zero cancellation). The consequence of this property is that the damping of the closed-loop poles is directly dependent on that of the open-loop system (nominal system). However, the

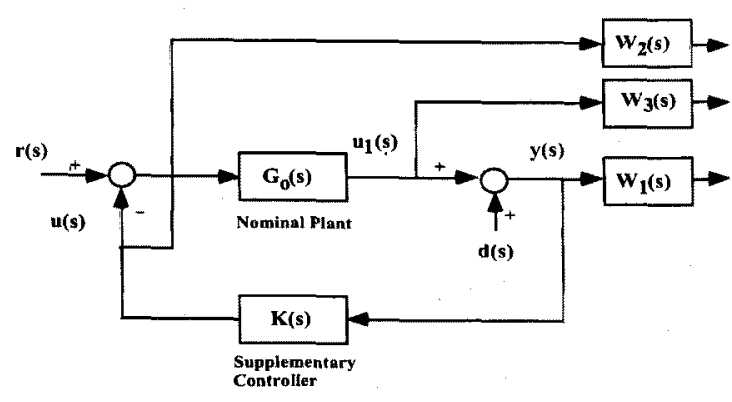

Fig. 4 Configuration for the damping control problem 
damping of the open loop system is usually unsatisfactory. Moreover, the cancellation of lightly damped poles can lead to poor robust stability and robust performance properties. When lightly damped poles are canceled, the controller cannot increase the damping of these modes and hence, the time response of the closedloop system will present lightly damped oscillations at the resonant frequencies.

\section{Controller Design}

This section deals with the design of one inputs $\left(I_{m}\right)$, one output SVC Supplementary Controller using a combination of additive and multiplicative uncertainty representations. The objectives of the design are to enhance the damping of weakly damped modes in a single machine connected to an infinite bus and to investigate the influence of uncertainty representation on the performance of the controller.

\subsection{Uncertainty Representation}

For a combination of additive and multiplicative uncertainty representation, the set of perturbed plants $\mathrm{G}(\mathrm{s})$ may be defined as:

$$
G(s):=\left\{\left[I+\Delta_{M}(s) W_{3}(s)\right] G_{o}(s)+\Delta_{A}(s) W_{2}(s)\right\}
$$

where $\Delta_{A}(s)$ and $\Delta_{M}(s)$ denote additive and multiplicative uncertainty, respectively and satisfy :

$$
\left\|\Delta_{A}(s)\right\|_{\infty} \leq 1 \text { and }\left\|\Delta_{M}(s)\right\|_{\infty} \leq 1
$$

First, this type of uncertainty representation allows the specification of both the control sensitivity function $R(s)$ and the complementary sensitivity function $T(s)$ at a time. The minimization of $R(s)$ and $T(s)$ leads to the improvement of the system's robustness to additive and multiplicative uncertainties, respectively. Furthermore, the minimization of $R(s)$ is useful to limit the amplitude of the control signal (the output signal of SVC supplementary controller) to its physical bounds while small $T(s)$ is necessary for high frequency disturbance reduction.

Secondly, this uncertainty representation can treat the system's uncertainties more flexibly.

\subsection{Design Specifications and Choice of the Weights}

The proposed Controller is required to maintain the performance and the robustness of the system in the presence of uncertainties as described in (7). Besides, robust performance should also be maintained in the presence of disturbances such as faults in the system, etc. And also, it has to be taken into account that the difference between the simplified and the higher order model can be assumed as uncertainties, which used in the design stage and the verification stage, respectively. This implis that a tight setting of $W_{2}$ and $W_{3}$ is quite important to increase the performance as well as robustness of the controller.

For good robustness against additive and multiplicative uncertainty, the bandwidth of the transfer function from d to ul ( Figure 4) is limited to $20 \mathrm{rad} / \mathrm{sec}$. Moreover, $W_{2}(s)$ is chosen such that the amplitude of the control signal being applied to the plant input is reduced. The sensitivity function $S(s)$ is required to be small up to the natural frequency $(4.85 \mathrm{rad} / \mathrm{s})$.

For good comprimise between performance and complexity of the controller is given by performance weighting function, $W_{l}(s)$, of the form:

$$
W_{1}(s)=\frac{T_{1} s+1}{T_{2} s+A}
$$

It can be analyzed that A determines the steady-state error ( $A$ will be zero if zero steady state error is required); $T_{1}$ approximately determines the bandwith $\left(\omega_{b} \approx 1 / T_{1}\right)$ and hence the rising time and settling time; where the ratio $T_{2} / T_{1}$ is associated with performance requirements against high-frequency noise.

To satisfy our design requirements, the following weighting functions $W_{1}(s), W_{2}(s)$ and $W_{3}(s)$ were chosen. These weights assure a good time response and shorter settling time.

$$
\begin{aligned}
& W_{1}=\frac{100(s+2000)}{(s+10)} \\
& W_{2}=\frac{100\left(0.1 s^{2}+100 s+1\right)}{\left(0.1 s^{2}+1.1 s+1\right)} \\
& W_{3}=\frac{0.01\left(0.1 s^{2}+10 s+1\right)}{\left(0.1 s^{2}+1.1 s+1\right)}
\end{aligned}
$$

\subsection{Bilinear Transform}

Furthermore, to prevent the pole-zero cancellation and improve the system's dynamic and transient stability, bilinear transformation method have been applied to the plant in our design. Letting the new complex plane be $\tilde{s}$-plane, the transformation is written as follows:

$$
s=\frac{\left(\tilde{s}+p_{1}\right)}{\left(1+\tilde{s} / p_{2}\right)}
$$

By setting the parameters of the bilinear transform $p_{1}<0$ and $p_{2}<0$ appropriately, poorly damped poles are moved away from the $\mathrm{j} \omega$ axis and places in right-half plane. In our design $p_{1}$ was chosen to be -0.3 and it is mainly used to shift the lightly damped poles of the nominal system into the right-half plane, i.e., to control the locations of the light damped modes. On the other hand, $p_{2}=-200$, was used to prevent a non-minimum phase controller (zeros in right-half plane). By using the bilinear transform, we can shift the poles and zeros away from the $\mathrm{j} \omega$-axis. After the controller is computed, the inverse bilinear transform is used to map the controller back to the original s-plane. It should be pointed out that the resulting controller is suboptimal for the actual system, but it can prevent the pole zero cancellation and can improve the damping of the poorly damped poles.

\subsection{Controller}

The optimal solution of equation (5) has yield a controller of $5^{\text {th }}$ order.

$$
K(\mathrm{~s})=\frac{3.9 e 2 \mathrm{~s}^{5}+1.4 e 5 \mathrm{~s}^{4}+1.3 e 7 \mathrm{~s}^{3}-4.7 e 6 \mathrm{~s}^{2}-8.9 e 7 \mathrm{~s}-2.7 e 7}{\mathrm{~s}^{5}+1.5 e 2 \mathrm{~s}^{4}+4.7 \mathrm{~s}^{3}-1.5 e 4 \mathrm{~s}^{2}+8 e 5 \mathrm{~s}+2.5 e 5}
$$

We can clearly notice an improvement in the system damping by this controller. The bode plots of open- and closed-loop systems are shown in figure 8 . It can be seen clearly that the peak value of the closed-loop system in the oscillation frequency is smaller than that of open-loop system. The damping ratios of the open and closed-loop system are listed in Table 1. From this table, it is clear that the desired damping of the dominant oscillation mode is achieved. 
Table 1 Damping ratio of Open-loop and Closed-loop System

\begin{tabular}{|l|c|c|c|}
\hline \multirow{2}{*}{ System Configuration } & \multicolumn{3}{|c|}{ Local Moded Damping Ratio } \\
\cline { 2 - 4 } & Open loop & $\begin{array}{c}\mathrm{H}_{\infty} \\
\text { Controller }\end{array}$ & $\begin{array}{l}\text { Phase Lead } \\
\text { Controller }\end{array}$ \\
\hline Nominal System & 0.0009 & 0.238 & 0.205 \\
\hline Reverse Flow & 0.0023 & 0.168 & -0.456 \\
\hline $\begin{array}{l}\text { Increasing Transfer } \\
\text { Power }\end{array}$ & 0.0004 & 0.065 & 0.017 \\
\hline $\begin{array}{l}\text { Damping Parameter } \\
\mathrm{D}=-5\end{array}$ & -0.0977 & 0.061 & -0.006 \\
\hline
\end{tabular}

\section{Performance Evaluation}

\subsection{Time domain responses equipped with Supplementary Controller of SVC}

In order to evaluate the damping effect of the SVC, we compare two types of $\mathrm{H}_{\infty}-\mathrm{SVC}$ supplementary controller: the standard and the improved controllers have been designed. The results are also compared with those of the conventional supplementary controller which consist of a wash-out and two phase-lead blocks. Parameters for the conventional controller are tuned optimally by trial and error manners to have maximum robustness and performance for the pre-specified set of system conditions.

Figure 5 shows the transmission line current output time responses of the closed-loop nominal system after a small disturbance is applied by a sudden variation to $V_{\text {ref. }}$. It can be seen that the responses of the system equipped with the improved SVC supplementary controller is well damped as compared with thoseof the conventional SVC supplementary and the standard $\mathrm{H}_{\infty}$-SVC supplementary controller. On the other hand, it can be observed that due to the pole-zero cancellation problems, the standard $\mathrm{H}_{\infty}-\mathrm{SVC}$ supplementary controller was unable to damp the oscillations in the nominal system as expected. i.e, the time response is not improved compared with open-loop system (without supplementary controller). This $\mathrm{H}_{\infty}-\mathrm{SVC}$ supplementary controller is not practically useful.

To prove the transient stability, the gain of the exciter was increased from 20 to 100 . Figure 6 shows the system responses of the transmission line current after one transmission line outage. In this case, the system oscillations are more pronounced and the controllers should be able to maintain the stability of the system. Again, the proposed SVC supplementary controller gives satisfactory performance as compared to the other designed SVC supplementary controller. Figure 7 shows good response of the closed loop system with the robust controller subject to increasing of the power transferring capacity from $200 \mathrm{MW}$ until $320 \mathrm{MW}$.

The simulation results have indicated that the robust controller can maintain the system stability under all realistic change in system conditions and can provide greater damping than the conventional supplementary controller. The designed controller satisfies all the design objectives stated before.

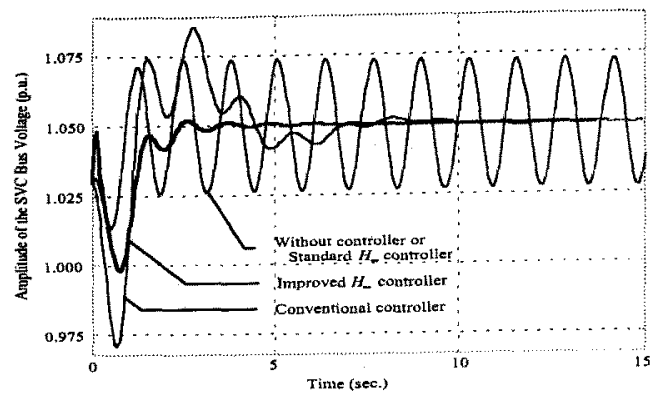

Fig. 5a Responses of Voltage Bus SVC under Nominal Operating Condition

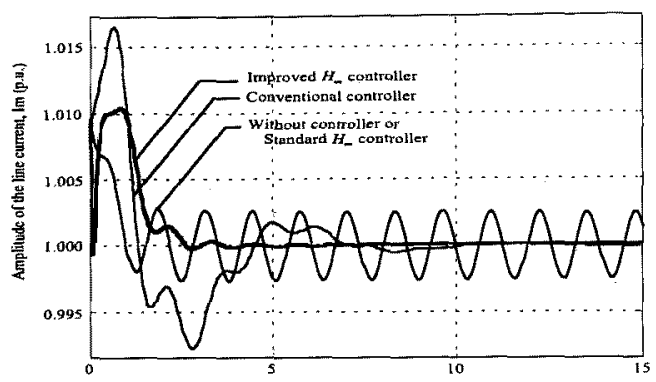

Fig. 5b Responses of Transmission Line Current, Im, under Nominal Operating Condition

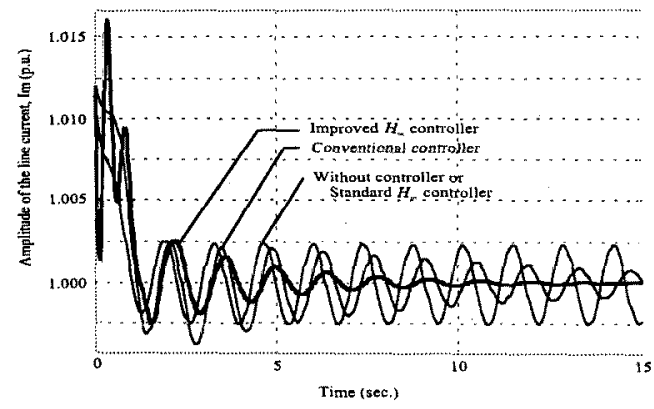

Fig. 6 Responses of the system for One Transmission Line outage and High AVR GAin

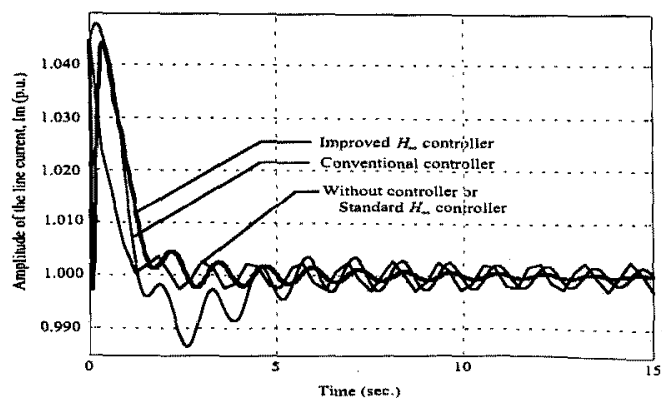

Fig. 7 Responses of the system subject to increasing transfer power 


\subsection{Disturbances Attenuation and Robustness Analysis}

Under the nominal operating condition, for the system with and without auxiliary stabilizing signal of SVC controller, the following observations are noted. The original system is stable with very low damping, which is far from satisfactory. By eigenvalue analysis, it is shown that the dominant poles are $-4.58 \mathrm{e}-$ $03 \pm j 4.85$. The damping ratio of this mode, $\zeta$, is 0.000944 , and the frequency of this swing mode is $4.85 \mathrm{rad} / \mathrm{s}$ or $0.77 \mathrm{~Hz}$. After applying the improved $\mathrm{H}_{\infty}$ - and the conventional SVC supplementary controller, the dominant poles of the closed-loop system are now $-1.35 \pm \mathrm{j} 5.51$ with a damping ratio $\zeta=0.238$ and $-0.97 \pm \mathrm{j} 4.64$ with a damping ratio $\zeta=0.205$, respectively.

Figure 8 depicts the singular value Bode plots of the closed loop system from disturbance $V_{\text {ref }}$ to the output $y$. It is obvious that the frequency response of the system equipped with the standard $\mathbf{H}_{x}-$ SVC supplementary controller peaks at the dominant poorly damped modes, same as open-loop system. This means that the disturbances are not attenuated by this supplementary controller. On the other hand, the conventional SVC supplementary controller and the improved $\mathrm{H}_{\infty}-\mathrm{SVC}$ supplementary controller have noticeably reduced this peak, i.e., they can attenuate the system oscillations. However the peaks of the improved $\mathrm{H}_{\infty}-\mathrm{SVC}$ supplementary controller is smaller than the conventional one. The smaller the peak, the better disturbance will be attenuated.

The stability margin in the face of additive and multiplicative plant perturbations can be measured using the inverse of the $H_{\infty}$. norm of the control sensitivity matrix $R(s)$ and the complementary sensitivity matrix $\mathrm{T}(\mathrm{s})$, respectively. Let $\beta$ and $\mu$ be defined as follows :

$$
\beta=\|R(j \omega)\|_{\infty}^{-1} \text { and } \mu=\|T(j \omega)\|^{-1}
$$

The values of these parameter as well as the ramping ratio of the dominant closed-loop modes are given in Table 2 . The bigger $\beta$ and $\mu$, the larger are the stability margin in the presence of additive and multiplicative uncertainties, respectively. It can be observed from Table 2 that the improved SVC Supplementary controller is more robust than the conventional SVC supplementary controller.

\section{Conclusion}

In this paper, two types of $\mathrm{H}_{\infty}-\mathrm{SVC}$ supplementary controllers: the standard and the improved are compared with the conventional SVC supplementary controller. The performance of the closed-loop systems are evaluated by eigenvalue analysis and numerical simulations. From these studies, we can conclude the followings:

(1) With proper formulation and using bilinear transform, the supplementary controller is capable of providing sufficient damping to the system oscillatory mode.

(2) The model variations caused by changes in system conditions, such as inaccuracies in the parameter values, change in operation conditions, can successfully be treated.

(3) The $\mathrm{H}_{m}$ designed controller has better performance and more robustness as compared to the $\mathrm{H}_{-}$standard supplementary controller and the optimally tuned conventional supplementary controller.

(Manuscript received October 4, 1999, revised February 25, 2000)

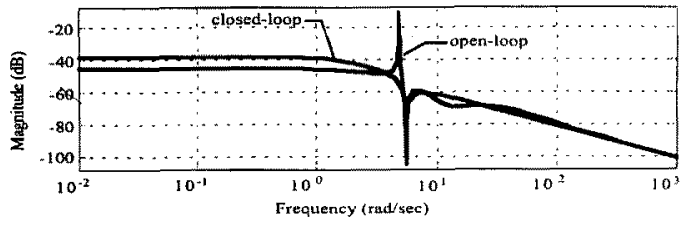

Fig. 8 Singular Value Bode Plots of $G_{o}(s)\left(I+G_{o}(s) K(s)\right)^{t}$

Table 2. Stability Margins and Dominant Damping Ratio

\begin{tabular}{|l|c|c|c|}
\hline & $\begin{array}{c}\text { Stability } \\
\text { Margin, } B\end{array}$ & $\begin{array}{c}\text { Stability } \\
\text { Margin }, \mu\end{array}$ & $\begin{array}{c}\text { Damping } \\
\text { Ratio }\end{array}$ \\
\hline Open loop & - & - & 0.0009 \\
\hline With Conventional Contr. & 0.005 & 0.927 & 0.205 \\
\hline${\text { With } H_{\infty} \text {-Approach Contr. }}$ & 0.030 & 1.013 & 0.238 \\
\hline
\end{tabular}

\section{References}

[1] K.R. Padivar, and R.K. Varma, "Damping Torque Analysis of Static Var System Controllers", IEEE Trans. Power Systems, Vol. 6, No. 2, pp. 458-465, May 1991

[2] J.R. Smith, D.A. Pierre, D.A. Rudberg, I. Sadighi, and A.P. Johnson, "An Enhanced LQ Adaptive Var Unit Controller for Power system Damping", IEEE Trans. Power Systems, Vol. 4, No. 2, pp 443-451, May 1989.

[3] J.R. Smith, D.A. Pierre, I. Sadighi, M.H. Nehrir, and J.F. Hauer," A supplementary Adaptive Var Unit Controller for Power System Damping", IEEE Trans. Power Systems, Vol. 4, No. 3, pp. 1017-1023, August 1989.

[4] R.Y. Chiang and M.G. Sofonov, Robust Control Toolbox User's Guide, The Math works Inc., 1992.

[5] H. Kwakernak,"Robust Control and $\mathrm{H}_{\infty}$ OptimizationTutorial Paper", Automatika, Vol. 29, No. 2, pp. 255-273, 1993.

[6] K.Ohtuka, S.Yokogawa,"A $\mathrm{H}_{\infty}$ Optimal Theory Based Generator Control System", IEEE Trans. energy Conversion, Vol. 7, No. 1, pp. 108-113, March 1992.

[7] Y. Uriu, M. Ohasi, F. Koyanagi, "Stabilization for MultiMachine Power system by decentralized Generator Control Strategy Using $\mathrm{H}_{\infty}$ Control Theory.

[8] Q. Zhao, J. Jiang, " Robust SVC Controller Design for Improving Power System Damping", IEEE Trans. Power Systems, Vol. 10.4, Nov. 1995.

[9] H. Okamoto, A. Kurita, "A Method for SVC Damping Controller design using Robust Pole Assignment Method", Trans. IEE Japan, Vol. 117-B. No. 4, pp. 578-584,1997.

[10] T. Shigetoh, A. Yokoyama, "Relationship between robust Stability and Power system damping enhancement by excitation Control System of Adjustable-Speed Generator", Trans. IEE Japan, Vol. 118-B. No. 1, pp. 6-14,1998.

[11] K.A. Folly, N. Yorino and H. Sasaki, "An improved $\mathrm{H}_{\infty}$ Power system Stabilizer", Trans. IEE Japan, Vol. 116-B. No. 12 , pp. 1470-1477,1996. 
[12] K.A. Folly, N. Yorino and H. Sasaki, "Design Of $H_{\infty}$-PSS Using Numer-Denumerator Uncertainty Representation", IEEE Trans. Energy Conversion, Vol. 12.1, pp. 45-50, March 1997.

[13] EPRI Report, "Improved Static Var Compensator Control", EPRI TR-100696, Project 2707-01, June 1992.

[14] P.M. Anderson, A.A. Fouad, "Power System Control and Stability", 1977.

\section{Appendix}

\section{A. System's data}

Generator data (MVA base: 900$) \quad x d=1.8 \quad x q=1.7 \quad x d^{\prime}=0.3$ $\mathrm{xq}$ ' $=0.55 \mathrm{xd}$ ' $=0.25 \mathrm{xl}=0.2 \mathrm{ra}=0.0025$

Tdo'=8 tdo" $=0.03$ tqo" $=0.05 \mathrm{H}=6.5 \mathrm{D}=0.025$

Excitation Data : $\mathrm{Ka}=0.003 \mathrm{Ta}=0.05 \mathrm{Kf}=0.02 \mathrm{Tf}=0.8$

Network Data (MVA base: 100) : $r=0.0001 \mathrm{pu} / \mathrm{km} \mathrm{x}=0.001 \mathrm{pu} / \mathrm{km}$ b2 $=0.00175 \mathrm{pu} / \mathrm{km}$

Transformer Data (MVA base : 900 ) : $\mathrm{re}=0 \mathrm{xe}=0.15 \mathrm{pu}$

SVC Data : $\mathrm{Kr}=25 \mathrm{Tr}=1 \mathrm{~B}=0.222 \mathrm{~K}_{\mathrm{sL}}=0.05$ (Slope Setting)

load1: $882 \mathrm{MW}, 50$ MVAR ,load2 : $396 \mathrm{MW}, 50$ MVAR

\section{B. Higher order model of power system}

For verification process, higher order model is used to discribe the dynamic behavior of the power system. The synchronous machine is represented by $7^{\text {th }}$ order model, which the axis-currents have been chosen as state variables. Elements of the generator state vector are $\mathrm{x}_{\mathrm{g}}{ }^{\mathrm{T}}=\left[\Delta \mathrm{i}_{\mathrm{d}}, \Delta \mathrm{i}_{\mathrm{q}}, \Delta \mathrm{i}_{\mathrm{f}}, \Delta \mathrm{i}_{\mathrm{D}}, \Delta \mathrm{i}_{\mathrm{Q}}, \Delta \delta, \Delta \omega, \Delta \mathrm{E}_{\mathrm{fd}}\right.$, $\left.\Delta \mathrm{T}_{m}\right]^{\mathrm{T}}$. See reference [14]

The state vector corresponding to the static compensator module is $\mathrm{x}_{\mathrm{c}}^{\mathrm{T}}=\left[\mathrm{s} \mathrm{x}_{\mathrm{c} 2}, \Delta \mathrm{x}_{\mathrm{c} 3}, \Delta \mathrm{x}_{\mathrm{c} 4}, \Delta \mathrm{x}_{\mathrm{c} 5}\right]^{\mathrm{T}}$. The current through the static compensator inductor is a controlled variable. The corresponding state equations is

$$
\Delta i_{C}=\dot{x}_{c 2}=V_{0} \Delta B+B_{c} \Delta V
$$

$B_{c}$ is the steady state value which is the inverse of the static compensator inductance and corresponds to the thyristor firing angle $\alpha_{o}$. The small variation quantity $\Delta B$ im eq. (B1) is treated as a state variable. In Fig. B1 are shown the transfer function blocks related with the control operation of the Static Compensator. The variational quantity $\Delta B$ achieved as a consequence of controlled thyristor firing is labelled as a state variable $x_{c 4}$. The other state variables indicated are $\mathrm{x}_{\mathrm{c} 3}$ and $\mathrm{x}_{\mathrm{c} 5}$. The voltage variation at busSVC

, $\Delta V_{\text {bus } S V C}$, and $\mathrm{x}_{\mathrm{c} 3}$ are related as below

$$
\dot{x}_{c 3}=-x_{c 3}+\Delta V_{b u s s V C}
$$

where $\Delta V_{\text {busSVC }}=\Delta V$

And the state equation corresponding to the control configuration in Fig. B1 is

$$
\left[\begin{array}{ccc}
T_{m r} & 0 & 0 \\
0 & T_{t} & 0 \\
0 & 0 & T_{r}
\end{array}\right]\left[\begin{array}{l}
\dot{x}_{3} \\
\dot{x}_{4} \\
\dot{x}_{5}
\end{array}\right]=\left[\begin{array}{ccc}
-1 & 0 & 0 \\
0 & -1 & 1 \\
-K_{r} & 0 & -1
\end{array}\right]\left[\begin{array}{l}
x_{3} \\
x_{4} \\
x_{5}
\end{array}\right]+\Delta V
$$

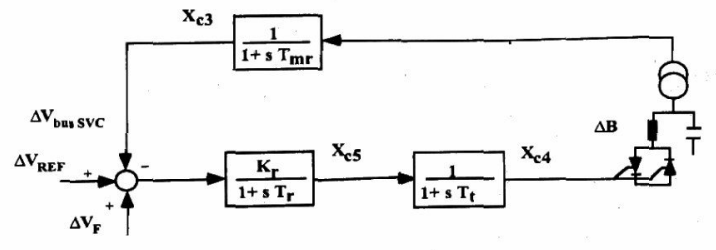

Fig. B1. Static Compensator control block diagram

$$
\begin{array}{ll}
\mathrm{T}_{\mathrm{mr}}: \text { Measuring delay time constant } \\
\mathrm{T}_{\mathrm{t}}: \text { Firing delay time constant } \\
\mathrm{K}_{\mathrm{r}}, \mathrm{T}_{\mathrm{r}}: \text { SVC main controller parameters }
\end{array}
$$

FERDI Armansyah (Student Member). He was born onNovember 14, 1965. He received the

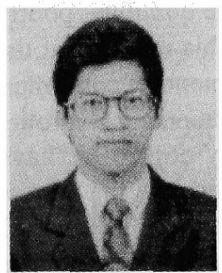
M.Sc degree from Technische Universiteit Delft, Holland in 1993. Presently he is preparing a $\mathrm{Ph}$. $\mathrm{D}$. on robust control and its application to power system dynamic stability at the Department of Electrical Engineering of Hiroshima University.

Naoto YORINO (Member). He was born on January 24, 1958. He received B.S., M.S., and Ph.D. degrees

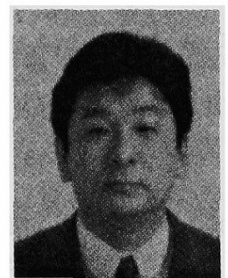
in electrical engineering in 1981, 1983 and 1987, respectively, all from Waseda University, Tokyo. Presently, he is an Associate Professor of Department of Electrical Engineering of Hiroshima University. He joined Fuji Electric Co.,Ltd., Japan from 1983 to 1984

He was a Visiting Professor at the McGill University, Canada from 1991 to 1992 . His research interests are mainly power system stability and control problem. Dr. Yorino ia a member of IEE of Japan.

Hiroshi SASAKI (Member). He was born on March 10,1941. He received B.S., M.S., and Ph.D. degrees in

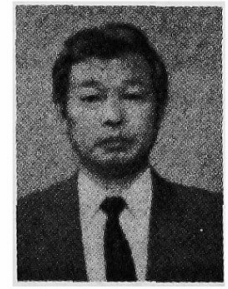
electrical engineering from Waseda University, Tokyo, in 1963, 1965 and 1979, respectively. He is a Professor of Department of Electrical Engineering of Hiroshima University. He was given Visiting Lecturer-ship from the University of Salford, Salford, England from 1971 to 1972.

He was a Visiting Professor at the University of Texas, Arlington from 1984 to 1985 . He has been studying various problems in power engineering field; especially expert system and neural network applications to power systems, etc. Dr. Sasaki is a member of the IEEE, CIGRE and several academic societies. 